\title{
OBSERVING MARS FROM AREOSTATIONARY ORBIT: BENEFITS AND APPLICATIONS
}

A White Paper submitted to the Planetary Science and Astrobiology Decadal Survey 2023-2032

\section{Primary authors}

Luca Montabone, Space Science Institute (USA) and National Space Science and Technology Center/United Arab Emirates University(UAE), 1montabone@ spacescience.org, +33 650243565 Nicholas Heavens, Space Science Institute (USA), nheavens@spacescience.org, +44 748160671

\section{Co-authors}

Jose L. Alvarellos, NASA ARC (USA) Michael Aye, LASP-CU Boulder (USA)

Alessandra Babuscia, JPL/Caltech (USA)

Nathan Barba, JPL/Caltech (USA)

J. Michael Battalio, Yale University

Tanguy Bertrand, NASA ARC (USA)

Bruce Cantor, MSSS (USA)

Michel Capderou, LMD/École Polytech. $(\mathrm{Fr})$

Matthew Chojnacki, PSI (USA)

Shannon M. Curry, UC Berkeley (USA)

Charles D. Edwards, JPL/Caltech (USA)

Meredith K. Elrod, Univ. of Maryland (USA)

Lori K. Fenton, SETI Institute (USA)

Robin L. Fergason, USGS (USA)

Claus Gebhardt, NSSTC/UAEU (UAE)

Scott D. Guzewich, NASA GSFC (USA)

Melinda A. Kahre, NASA ARC (USA)

Ozgur Karatekin, Royal Observatory (Belgium)

David M. Kass, JPL/Caltech (USA)
Robert Lillis, UC Berkeley (USA)

Giuliano Liuzzi, NASA GSFC (USA)

Michael A. Mischna, JPL/Caltech (USA)

Claire E. Newman, Aeolis Research (USA)

Maurizio Pajola, INAF-OAPD (Italy)

Alexey Pankine, SSI (USA)

Sylvain Piqueux, JPL/Caltech (USA)

Ali Rahmati, UC Berkeley (USA)

M. Pilar Romero-Perez, IMI-UCM (Spain)

Marc Sanchez-Net, JPL/Caltech (USA)

Michael D. Smith, NASA GSFC (USA)

Alejandro Soto, SwRI (USA)

Aymeric Spiga, LMD/Sorbonne Univ. (France)

Leslie Tamppari, JPL/Caltech (USA)

Joshua Vander Hook, JPL Caltech (USA)

Paulina Wolkenberg, INAF-IAPS (Italy)

Michael D. Wolff, SSI (USA)

Ryan C. Woolley, JPL/Caltech (USA)

Roland M. B. Young, NSSTC/UAEU (UAE)

"MANY MAY CONSIDER THE SOLUTION PROPOSED IN THIS DISCUSSION TOO FARFETCHED TO BE TAKEN VERY SERIOUSLY " - A.C. Clarke, 1945

A.C. Clarke wrote the above sentence in 1945, talking about geostationary "stations" [1]. Yet, the first geostationary communication satellite (Syncom 3) was launched in 1964, and about 400 orbit Earth today. Geostationary satellite imagery revolutionized Earth weather forecasting in the 1970s. The areostationary orbit is the Mars-equivalent of the geostationary one. This white paper has the objective to detail what scientific and operational applications would highly benefit from platforms in this type of orbit. The needs are compelling and the technology is mature to see the first areostationary satellites orbiting Mars in the next decade. Beyond Mars, planetostationary orbits in general can provide similar benefits for future planetary exploration.

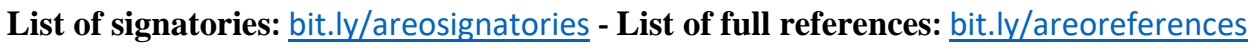

A portion of the writing and preparation of this White Paper was carried out at the JPL/Caltech,

July 15,2020 under a contract with the National Aeronautics and Space Administration (80NM0018D0004). 


\section{Introduction}

The equatorial, circular orbit in which the orbital period of the satellite matches the rotational period of the planet has long been recognized as a useful vantage point for observing a fixed region of a planet through the course of its day and facilitating communication across its surface. This type of orbit is called geostationary at Earth and areostationary at Mars. Any satellite orbit represents a tradeoff between observing locations globally, continuously, simultaneously, and/or closely. The polar Sun-synchronous orbit, for example, allows locations to be observed globally at fixed local times and low altitudes, but does not permit wide portions of the planet to be monitored continuously or simultaneously. The areostationary orbit trades low altitude and polar coverage for the ability to observe the same full-planet disk at all local times over the course of the Martian day, thus prioritizing continuous and simultaneous observations.

It is important to stress that satellites in areostationary orbit introduce monitoring to the observation paradigm for an extraterrestrial planet, so far only focused on mapping.

Low-altitude, planet-asynchronous orbits are ideal for global, high-resolution mapping of locations when one does not require the continuity and simultaneity of observations at each location; high-altitude, planet-synchronous orbits provide vantage points to monitor dynamical phenomena rapidly evolving in space and time. An areostationary platform, therefore, has distinct advantages for various areas of Martian science and exploration, described by this paper.

\section{Elements of areostationary orbit dynamics}

An areostationary satellite would orbit Mars in a circular and equatorial orbit with a semi-major axis of $\mathrm{as}_{\mathrm{s}}=20,428 \mathrm{~km}$ (altitude of $17,031.5 \mathrm{~km}$ ) to be at rest with respect to the rotating Mars with a period of one Martian sidereal day (sol): $\mathrm{P}=88,642.663 \mathrm{~s}$ (see [2] for a derivation of the orbit characteristics). The locations having zero tangential acceleration correspond to two stable longitudes $\left(17.92^{\circ} \mathrm{W}\right.$ and $\left.167.83^{\circ} \mathrm{E}\right)$ and two unstable longitudes $\left(75.34^{\circ} \mathrm{E}\right.$ and $\left.105.55^{\circ} \mathrm{W}\right)$ [3].

However, natural perturbations tend to move an areostationary satellite from its nominal station point. The dominant disturbing forces on the orbit are perturbations due to the nonspherical mass distribution of the Mars gravitational field, the gravitational attraction of the Sun, the moons Phobos and Deimos (the areostationary orbit lies between the nearly equatorial orbits of the two moons, having $a_{s}=9,376 \mathrm{~km}$ and $a_{s}=23,463.2 \mathrm{~km}$, respectively), and the Solar Radiation Pressure (SRP). These perturbations have been described and modeled in [3-6]. Here we simply note that the perturbations of the Mars gravitational field cause a change of the semimajor axis of the orbit that, in turn, induces a longitudinal drift; the attraction of the Sun and moons cause a change of the orbit's inclination and, consequently, latitudinal oscillations; SRP causes a change of the mean eccentricity vector that also contributes to the longitudinal drift. A satellite orbiting at a lower (higher) altitude than the nominal areostationary one would drift eastwards (westwards) about $1 \%$ sol every $38 \mathrm{~km}$ altitude difference. As with geostationary satellites, areostationary ones would therefore require station keeping maneuvers to counteract natural perturbations. With the exception of stable longitudes, on average, areostationary eastwest station keeping requires a lot more $\underline{\Delta v}$ than geostationary [3-4]; conversely, north-south station keeping requires less $\Delta \mathrm{v}$ because Mars's moons are smaller and the Sun is farther [5].

\section{Benefits of the areostationary orbit}

\section{$>$ 3.1. Continuous and simultaneous coverage}

The ideal areostationary orbit has its sub-spacecraft point fixed in longitude at the equator at a fixed altitude. If the orbit is maintained with station keeping maneuvers, it is possible to: 
1. Observe the same Martian disk to nearly

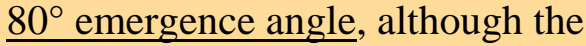
portion of the disk useful for quantitative observations might be limited to about $60^{\circ}$ (see Fig. 1);

2. Monitor the observed area continuously and simultaneously, which, in practice, means that data can be obtained at a sub-hourly cadence from all locations in the area at once;

3. Acquire observations throughout the full diurnal cycle.

One areostationary orbiter, alone, can focus the monitoring on a specific region of interest and complement other globalmapping orbiters (e.g. the "MAT" mission concept, [8]). Although the advantage of having the same view of Mars all the time is
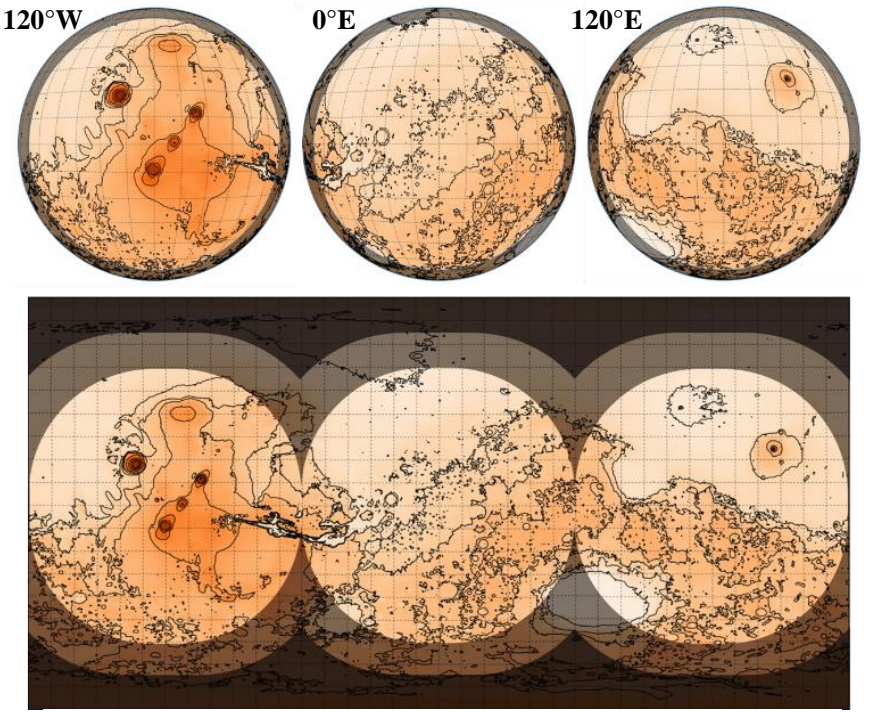

Figure 1: Three areostationary views and equivalent equirectangular projection of Mars topographic contours. The light grey band starts at $60^{\circ}$ emergence angle, the dark grey one starts at $75^{\circ}$ (poorly or not observed regions). lost if the orbit is not maintained with proper station keeping, there might be significant advantages in allowing a small longitudinal drift or latitudinal oscillation of a single quasiareostationary satellite. For instance, it could cover different longitudes or the polar regions.

Note that continuous and simultaneous monitoring has been explicitly identified as a high priority requirement by the Mars Exploration Program Analysis Group (MEPAG) to improve the spatiotemporal coverage of existing data (Goal II, Investigation A1) and to enable future human exploration missions (Goal IV, Investigation B3.1) [7].

\subsection{Quasi-global coverage (with an areostationary constellation)}

Quasi-global monitoring (excluding the polar regions) can be achieved by a constellation of areostationary platforms. The minimum number required for covering all equatorial longitudes is three (see Fig. 1). The required number to cover all longitudes in a continuous latitude band extending from nearly $80^{\circ} \mathrm{S}$ to nearly $80^{\circ} \mathrm{N}$ with some large overlapping in longitude is four ${ }^{1}$.

If partial coverage of the polar regions is also required, two of the four members in the constellation could be areosynchronous with a slight orbital inclination (e.g. $15^{\circ}-20^{\circ}$ ) to alternately lean towards the poles (in this case the sub-spacecraft point traces an " 8 " loop). Ideally, an areostationary constellation together with one or more satellites in low-altitude polar or highly elliptical orbits (e.g., "Molniya"-type orbit) would provide fully global, continuous, simultaneous, and close coverage (e.g. the "MOSAIC" mission concept, [9]).

\section{Scientific applications ${ }^{2}$ of the areostationary orbit}

\subsection{Atmospheric weather monitoring}

The significance of diurnal meteorological variability at Mars argues for orbital observations that span the diurnal cycle. The rapid dynamics of meteorological phenomena such as dust storms (typical timescale of a few hours) and water/ $\mathrm{CO}_{2}$ ice clouds (typical timescale of half an hour or less), together with their spatial extension (up to the planetary scale for dust events) and duration/repeatability, argues for continuous and simultaneous observations across the planet.

\footnotetext{
${ }^{1}$ Animation of a four-satellite areostationary constellation: $\underline{b i t . l y / a r e o a n i m a t i o n}$

${ }^{2}$ Table of related physical parameters, observable quantities, and available instruments: bit.ly/areotable
} 
Because of the approximately diurnal radiative relaxation timescale of its thin atmosphere, the diurnal cycle of insolation influences the weather on Mars even more than it does on Earth, where the equivalent timescale is about a month [10]. The lower atmosphere rapidly responds to solar radiation absorbed by a surface with varying albedo and elevation, and by atmospheric dust aerosol. It also responds to longwave cooling by water ice, and latent heating by $\mathrm{CO}_{2}$ ice. The mechanisms of dust lifting, vertical mixing, and scavenging are expected to have strong diurnal variability [11]. Dust lofted by storms can be transported from the surface to the mid-atmosphere in a matter of hours [12] and can substantially affect the upper atmosphere within a week or two [13-15]. The radiative forcing of surface variability and atmospheric aerosols is coupled through atmospheric heating/cooling, induced circulations, and cloud microphysics [16-17].

To date, Mars's weather has been monitored mainly from spacecraft in polar or quasi-polar orbits, which sample only a narrow range of local times, or several local times over multiple sols with discontinuous spatial coverage. When observations have been made and/or analyzed at different local times, a high magnitude of diurnal variability often has been found. Thick water ice clouds form at night in deep canyons and basins but rapidly evaporate in the early morning hours, while similarly thick clouds develop on high volcano summits during the day [e.g., 18]. Near-surface water vapor varies by an order of magnitude diurnally, suggesting significant exchange of water with the regolith [19]. Atmospheric dust loading can vary by up to an order of magnitude during the course of the day, particularly on the margins of large-scale dust storms [20-22]. The coverage provided by an areostationary platform is ideal for tracking and helping explain such extreme variability as well as investigating short duration phenomena that a polar orbiting spacecraft might miss entirely, e.g., local dust storms at low latitudes, which typically last less than a sol [23]. Areostationary imagery would improve counting these storms to quantify their hazard and contribution to the global dust budget as well as enable their life cycle to be studied in detail and compared with the much longer than diurnal life cycle of regional dust storms and global dust events. A network of areostationary platforms would monitor and better characterize the development of large-scale dust storms into extreme, global-scale events.

Areostationary imagery also could be used to quantify atmospheric winds using cloud tracking [24] and complement more systematic wind observations [25]. Cloud tracking would require additional information to constrain the altitude of the diagnosed winds (such as by wind and/or opacity measurements from a polar orbiter) but would yield measurements with high spatial and temporal resolutions in a limited vertical range throughout the observed domain. This would enable the diurnally variable global wind field [e.g., 26] and its spatial variability within weather systems to be identified, studied, and used for model validation [27].

\subsection{Space weather monitoring}

A monitoring station at an unobstructed vantage point that can observe the upstream solar wind (such is the case from areostationary orbit) is of critical interest for science and exploration. The high distance of an areostationary orbit from Mars also allows for globally mapping the escape of atmospheric species in response to changes in upstream drivers.

Unlike the Earth, which has a global magnetic field that protects its atmosphere from direct interaction with the solar wind, Mars's upper atmosphere is directly exposed to the solar wind and is heavily influenced by the dynamic nature of the upstream particles and fields emanating from the Sun and streaming throughout the heliosphere. This direct interaction can lead to heating, excitation, dissociation, ionization, and/or energization of the atmospheric species as well as atmospheric loss from the planet [28]. Therefore, monitoring the solar wind conditions is crucial for understanding the relationship between the upstream conditions at Mars and their 
effects on the atmosphere. Additionally, flares and coronal mass ejections at the Sun create solar energetic particle events that travel in the heliosphere with speeds much greater than the solar wind, and can penetrate Mars's bow shock. These energetic electrons and ions interact with the upper and lower atmosphere and can even reach the planet's surface [29]. They are important contributors to ionizing radiation that future orbital and surface human missions will experience.

Because it spends nearly $75 \%$ of its time outside the bow shock of Mars, an areostationary platform has a much less interrupted view of the upstream conditions compared to orbits at lower altitudes. Simultaneous in-situ measurements of solar wind conditions along with remote sensing of the atmosphere would enable study of the connection between solar energetic particle events and auroral activity at Mars [30]. The upstream measurements taken at an areostationary orbit can also be used in conjunction with other spacecraft data in order to establish a link between solar wind upstream parameters and space weather effects at Mars [31]. For the remaining 25\% of an areostationary orbit, observations of particles and fields in the magnetosheath and magnetotail would improve understanding of the physics involved in the interaction of the solar wind with Mars's bow shock. They also would allow for a better determination of processes that influence the effectiveness of the penetration of the bow shock by solar wind particles [32].

\subsection{Study of surface properties}

The interface between Mars's surficial regolith and ever-changing atmosphere is highly dynamic; it is shaped by a wide range of processes operating on timescales as short as a few seconds. A flurry of new science questions could be tackled with globally distributed remote sensing observations of the surface covering the full span of the diurnal cycle:

- Acquiring full diurnal surface temperature cycles would allow a deconvolution of regolith thermophysical properties at shallower depths, including the characterization of surficial duricrust layers, desert pavement, rock abundance, and dust layers with unprecedented accuracy. Diurnal ice cycles could also be characterized at night, verifying hypotheses regarding the location and variability of nighttime surface frosts. At the edge of the seasonal caps, diurnal vs. seasonal variability could be deconvolved and documented. High cadence thermal monitoring would help modelers to identify strong subsurface thermo-physical contrasts between water ice (or ice cemented soil) and dry regolith based on their effect on surface temperatures trends [33]. Such information could yield high and mid-latitudes shallow water ice deposit maps -valuable for In Situ Resource Utilization- at medium spatial resolution, but with unprecedented vertical resolution within the first meter of regolith;

- Integrated surface reflectance observations would help determine the energy and mass balance of icy surfaces at subpolar and mid-latitudes with global climate impact. Repeated seasonal observations under similar illumination conditions throughout the year would allow the tracking of subtle changes associated with surface dust movement and tie them to the replenishment or depletion of surface reservoirs. The variation with time of surface properties has been identified as most useful to determine dust abundance and mobility [11];

- The areostationary orbit is beyond the orbit of Phobos (which has an orbital period of 8h), so thermophysical and spectroscopic characterization of its leading and trailing hemispheres would be regularly possible under identical observational conditions. Imaging can contribute to quantify the thermal inertia of this tidally locked body, the mineralogical differences between the red unit [34] and the blue unit deposits [35], and the likely differential evolution of its hemispheres. Deimos orbits just outside the areostationary orbit. Its sub-Mars hemisphere can be observed at a wide range of local times with instruments that point away from Mars, yielding information on surface rock abundance, roughness, and grain sizes. 


\section{Operational applications of the areostationary orbit}

\subsection{Weather forecasting}

Areostationary observations would revolutionize Mars weather forecasting as geostationary satellite imagery did for Earth weather forecasting in the 1970s. The quasi-global, continuous, and simultaneous view that observations from an areostationary network provide would allow forecast initialization from atmospheric states that properly account for teleconnections, largescale weather and dust events, tides, and other diurnally varying, global-scale phenomena.

Weather forecasting at Mars is required to protect assets during Entry, Descent, and Landing (EDL) and on the surface, and is recognized as a high priority for enabling human exploration [7: IV.B3.1]. Accurate predictions of the onset and development of dust storms have proven to be particularly difficult to obtain [36]. A good forecast model and accurate initial conditions enable high-quality weather forecasting. The requirements to achieve the former are discussed in [11]. The latter requires comprehensive observations of the atmosphere, combined with a model using data assimilation, a technique that also fills in gaps where observations are sparse, and retrieves unobserved quantities. Data assimilation for Mars is continually being improved [37-39], but suffers from a lack of systematic observations. On Earth, due to the chaotic nature of the general circulation at all scales, the predictability horizon of a forecast is closely related to the accuracy of the initial condition [40]. Mars's atmosphere is less chaotic-more predictable- at certain times of the year [41], but is chaotic on inter-annual scales -global dust events occur in some years, but not in others [42]. Accurate initial conditions are still required to improve predictions and to minimize the effects of model biases [43]. The more complete the orbital and possibly surface observational networks, the more accurate the initial condition, the more skilled the forecast.

\subsection{Telecommunication}

Areostationary relay satellites could advance telecommunication capabilities for future robotic and human Mars missions far beyond current capabilities [44-46]. They are also ideal to support the communication requirements of a future generation of small, low-cost Mars science orbiters.

Today, a fleet of NASA and ESA Mars science orbiters equipped with UHF proximity link payloads provides a relay service for landers and rovers on the Martian surface and enhances the amount of data they can transmit. However, the low altitude science orbits of these satellites allow only intermittent contact opportunities for surface assets; for instance, NASA's Mars Reconnaissance Orbiter typically flies over the Curiosity rover just twice per sol, with each overflight lasting only $\sim 10 \mathrm{~min}$. While the short slant range to these low orbits enables simple, omnidirectional links with data rates of up to $2 \mathrm{Mb} / \mathrm{s}$, the limited contact times reduce total data return to $<1 \mathrm{~Gb} / \mathrm{sol}$, and constrain the local times of the available communication windows.

For a given user on the surface, an areostationary relay orbiter would always be in view, offering communication to/from Earth throughout the Martian sol -even at times when Earth is not in view from that specific surface location on Mars (with the exception of short-duration, seasonally-occurring occultations of no more than 80 minutes/sol). With round-trip light times of only about 10-40 minutes, one can then envision closing multiple Earth-Mars planning cycles in a single sol, transforming the way we interact with landed Mars spacecraft -or human crews.

At the higher areostationary orbit, however, $1 / \mathrm{R}^{2}$ space losses $-\mathrm{R}$ being the distance between transmitter and receiver- become one to three orders of magnitude larger than at the low orbits of current satellites. To address this, areostationary relay architectures will need to use higherfrequency bands, and directional proximity links between the user and the relay orbiter. These directional links can achieve extremely high relay data rates. Assuming a $1 \mathrm{~m}$ receiver antenna on the orbiter, a small X-band (8-GHz) communication system with $15 \mathrm{~W}$ transmit power and a 
$30 \mathrm{~cm}$ directional antenna -similar to what the Curiosity rover uses to communicate back to Earth at data rates of just $\sim 1 \mathrm{~kb} / \mathrm{s}$ - pointing up to an areostationary relay satellite would achieve data rates of $\sim 5 \mathrm{Mb} / \mathrm{s}$. Raising the relay link frequency to Ka-band (32 GHz) would further increase relay data rates up to $\sim 70 \mathrm{Mb} / \mathrm{s}$. Combining higher data rates and continuous availability translates into orders-of-magnitude increase in potential data return from the Martian surface.

Areostationary relay orbiters are also ideally located to offer excellent visibility to satellites in low-altitude science mapping orbits, particularly low-cost SmallSats [47]. An areostationary platform can carry a large communication payload that can be shared across several small platforms, thus minimizing their communication requirements and, by consequence, costs.

\subsection{Computational resources}

A number of important science questions about Mars require aggregation and combination of large amounts of data -including temporally dense imagery, seismic, radar, or spectral data- and could be greatly enhanced by processing and storage on an areostationary orbiter [48-49].

An areostationary orbiter is an ideal support mothership for constellations of both smaller satellites and landed assets. Such an orbiter, in direct and constant communication with a rover, could provide computational resources for map reading or image processing to support autonomous driving [50] and reduce rover onboard resources. It can also serve as a "Mars-local cache", taking up the mundane data management, and freeing time, energy, and computational resources for the landed asset to conduct science activities [51]. In addition, it could serve as a reserve of compute or data storage, to alleviate risk or cost to landed assets. [52].

\subsection{Navigation resources}

Areostationary platforms can be part of a Global Navigation Satellite System (GNSS), which would greatly improve mission safety and reliability for sustainable, long-term exploration [53].

A GNSS would provide surface and orbiting assets with the benefits of i) precise real-time autonomous positioning for fixed or moving surface assets, ii) precise real-time autonomous navigation during all mission phases around Mars, and iii) accurate timing available planet-wide.

\section{Conclusions and recommendations}

Areostationary orbiters are innovative platforms for space-based Mars science, from its moons to its sub-surface. Equally important, the first areostationary orbiter will blaze a trail for the orbital infrastructure that will enable humans to explore Mars's surface and sub-surface safely and efficiently. In order to achieve truly global coverage and synergistic perspective, such platforms can work in conjunction with, or as a complement to, satellites in polar or eccentric low-altitude orbits. They will also be platforms for testing general planetostationary satellite operations away from Earth, a concept possibly applicable to several other planetary bodies in the Solar System.

In this White Paper, we have described the benefits of having satellites in areostationary orbit around Mars, and the applications for science and exploration (both robotic and human):

- They provide continuous and simultaneous coverage of locations within the same Martian disk to nearly $80^{\circ}$ emergence angle, and can provide quasi-global coverage (with the exception of polar regions) if flown as a constellation of at least three platforms;

- Areostationary satellites can capture the full range of meteorological variability on timescales faster than the daily to fortnightly repeat cycle typical of other orbital platforms. Examples include the formation of local dust storms, the expansion of regional dust storms into global events, and the diurnal cycle of water ice clouds on high mountains and deep canyons alike;

- The areostationary orbit lies outside Mars's bow shock, enabling measurement of the timevariable forcing that originates in the solar wind at sub-hourly time scales. Simultaneous 
observations of the lower atmosphere and the space environment allow characterization of the thermosphere-ionosphere response to dust storms from below and solar flares from above;

- By observing changes in surface temperature and optical properties over diurnal, seasonal, and multiannual timescales, this type of platform allows continuous monitoring of the surface distribution and temporal evolution of aeolian materials, surface frosts, and lower latitude surface ice. The areostationary regime also allows for frequent imaging of Mars's moons;

- We expect that areostationary observations of atmospheric variables -particularly those collected by a constellation with quasi-global coverage- will enable weather forecasting when used for data assimilation in global and mesoscale atmospheric models;

- Several studies have highlighted the benefits of satellites operating in areostationary orbit for relaying communications from robotic and human missions on the Martian surface, allowing continuous streaming of data and anytime uplink of command sequences. They also represent ideal platforms for on-orbit computing to minimize the need to deliver computing resources to surface assets, and for integrating a future Mars Global Navigation Satellite System.

We recommend that areostationary orbiters be considered and prioritized by NASA and other space agencies in any architecture studied or developed for Mars in the next decade, including where the focus is exploring the surface and sub-surface in situ by robotic and human missions. We also recommend that the development of technologies areostationary orbiters can benefit from (e.g. optical communication) continue in the next decade. Finally, we encourage the study of the possible applications of planetostationary orbits for exploration outside the Mars system.

\section{References}

${ }^{\mathbf{1} C l a r k e} 1945$ Wireless World 51 305. ${ }^{2}$ Capderou 2005 Springer Verlag 978-2-287-21317-5. ${ }^{3}$ Silva \& Romero 2013 PSS 87 14. ${ }^{4}$ Alvarellos 2009 J. Astronaut. Sci. 57 701. ${ }^{\mathbf{5}}$ Romero+ 2015 J. Guid. Control Dyn. 38 2223. Romero \& Antolín 2014 Acta Astronautica 10227. ${ }^{7}$ Banfield+ 2020 MEPAG Science Goals. ${ }^{8}$ Montabone+ 2018 ICubeSat 2018.A.2.4. ${ }^{9}$ Lillis+ 2020 38 ${ }^{\text {th }}$ MEPAG meeting. ${ }^{10}$ Read+2015 Rep. Prog. Phys. $78125901 .{ }^{11}$ Newman+ 2020 White Paper PSA Decadal Survey. ${ }^{\mathbf{1 2}}$ Heavens+2019 JAS 76 3299. ${ }^{\mathbf{1 3}}$ Chaffin+ 2017 Nat. Geosci. 10174. ${ }^{14}$ Heavens+ 2018 Nat. Astron. 2 126. ${ }^{15}$ Girazian+ 2020 JGR Plan. 125 e2019JE006092. ${ }^{16}$ Michaels+ 2006 GRL 33 L16201. ${ }^{17}$ Neary+ 2019 GRL 47 e2019GL084354. ${ }^{\mathbf{1 8}}$ Glenar+ 2003 Icarus 161 297. ${ }^{19}$ Zent+ 2016 JGR Plan. 121 626. ${ }^{20}$ Wolkenberg \& Giuranna 2020 Icarus 113823. ${ }^{21}$ Kleinboehl+ 2020 JGR Plan., e2019JE006115. ${ }^{22}$ Montabone+ 2020 JGR Plan., e2019JE006111. ${ }^{\mathbf{2 3}}$ Wang \& Richardson 2015 Icarus 251 112. ${ }^{\mathbf{2 4}}$ Wang \& Ingersoll 2003 JGR 108 5110. ${ }^{\mathbf{2 5}}$ Guzewich+ 2020 White Paper PSA Decadal Survey. ${ }^{\mathbf{2}}$ Wilson \& Hamilton 1996 JAS 53 1290. ${ }^{27}$ Menzel 2001 BAMS 82 33. ${ }^{\mathbf{2 8}}$ Chassefière+ 2004 PSS 52 1039. ${ }^{29}$ Jakosky+ 2015 Science $3506261 .{ }^{30}$ Schneider+ 2018 GRL $457391 .{ }^{31}$ Lee+ 2017 JGR 122 2768. ${ }^{32}$ Kallio \& Janhunen 2001 JGR $1065617 .{ }^{33}$ Piqueux+ 2019 GRL 46 14290. ${ }^{34}$ Pajola+ 2013 ApJ 777 127. ${ }^{35}$ Pajolat 2018 PSS 154 63. ${ }^{\mathbf{3 6}}$ Montabone \& Forget 2018 ICES 281. ${ }^{\mathbf{3 7}}$ Lewis \& Read 1995 ASR 169. ${ }^{38}$ Greybush+2012 JGR 117 E11008. ${ }^{\mathbf{3 9}}$ Navarro+ 2017 ESS 4 690. ${ }^{\mathbf{4 0}}$ Lorenz 1969 BAMS 50345. ${ }^{\mathbf{4 1}}$ Newman+ 2004 QJRMS $1302971 .{ }^{\mathbf{4 2}}$ Pankine \& Ingersoll 2004 Icarus 170 514. ${ }^{\mathbf{4 3}}$ Rogberg+ 2010 QJRMS 136 1614. ${ }^{\mathbf{4 4}}$ Breidenthal+2018 SpaceOps Conf. 2426. ${ }^{\mathbf{4 5}}$ Edwards+ 2016 Proc. IEEE Aerosp. Conf. ${ }^{\mathbf{4 6}}$ Lock+2016 Proc. IEEE Aerosp. Conf. ${ }^{\mathbf{4 7}}$ Barba+ 2020 White Paper PSA Decadal Survey. ${ }^{\mathbf{4 8}}$ March \& Casler 2020 Acta Astronautica $16831 .{ }^{\mathbf{4 9}}$ Vander Hook+ 2020 Proc. IEEE Aerospace Conf. ${ }^{\mathbf{5 0}}$ Higa+ 2020 IEEE Robot Autom. Lett. $43876 .{ }^{\mathbf{5 1}}$ Estlin+2012 ACM Trans. Intell. Syst. Technol. 3 3. ${ }^{\mathbf{5 2}}$ Vander Hook+ 2019 ICAPS 29 707. ${ }^{\mathbf{5 3}}$ Pirondini \& Fernandez 2003 ESA Executive Summary. 